\title{
Striatal Dysfunctions Associated with Mitochondrial DNA Damage in Dopaminergic Neurons in a Mouse Model of Parkinson's Disease
}

\author{
Alicia M. Pickrell, ${ }^{1 \star}$ Milena Pinto, ${ }^{2 *}$ Aline Hida, ${ }^{2}$ and Carlos T. Moraes ${ }^{1,2,3}$ \\ ${ }^{1}$ Neuroscience Graduate Program and Departments of ${ }^{2}$ Neurology and ${ }^{3}$ Cell Biology and Anatomy, Miller School of Medicine, University of Miami, Miami, \\ Florida 33136
}

Parkinson's disease (PD) is one of the most common progressive neurodegenerative disorders, characterized by resting tremor, rigidity, bradykinesia, and postural instability. These symptoms are associated with massive loss of tyrosine hydroxylase-positive neurons in the substantia nigra (SN) causing an estimated $70-80 \%$ depletion of dopamine (DA) in the striatum, where their projections are located. Although the etiology of PD is unknown, mitochondrial dysfunctions have been associated with the disease pathophysiology. We used a mouse model expressing a mitochondria-targeted restriction enzyme, PstI or mito-PstI, to damage mitochondrial DNA (mtDNA) in dopaminergic neurons. The expression of mito-PstI induces double-strand breaks in the mtDNA, leading to an oxidative phosphorylation deficiency, mostly due to mtDNA depletion. Taking advantage of a dopamine transporter (DAT) promoter-driven tetracycline transactivator protein (tTA), we expressed mito-PstI exclusively in dopaminergic neurons, creating a novel PD transgenic mouse model (PD-mito-PstI mouse). These mice recapitulate most of the major features of PD: they have a motor phenotype that is reversible with L-DOPA treatment, a progressive neurodegeneration of the SN dopaminergic population, and striatal DA depletion. Our results also showed that behavioral phenotypes in PD-mito-PstI mice were associated with striatal dysfunctions preceding SN loss of tyrosine hydroxylase-positive neurons and that other neurotransmitter systems [noradrenaline (NE) and serotonin (5-HT)] were increased after the disruption of DA neurons, potentially as a compensatory mechanism. This transgenic mouse model provides a novel model to study the role of mitochondrial defects in the axonal projections of the striatum in the pathophysiology of PD.

\section{Introduction}

Parkinson's disease (PD) is one of the most common age-related, motor-deteriorating, progressive neurodegenerative diseases. Due to the loss of control over voluntary movements, resting tremor, postural instability, bradykinesia, and rigidity are the four cardinal signs of the disease. This is caused by the loss of a subpopulation of dopaminergic neurons of the substantia nigra (SN) that project their axons to the striatum with consequent dopamine depletion in this region. Neurons of the ventral tegmental area (VTA) that share very similar characteristics with those in the SN and are anatomically close are relatively spared in the disease.

Disruptions in oxidative phosphorylation (OXPHOS) have been associated with the disease and are believed to act as primary or secondary contributors to neuronal loss in PD. PD postmor-

\footnotetext{
Received Sept. 24, 2011; revised 0ct. 18, 2011; accepted Oct. 20, 2011.

Author contributions: A.M.P., M.P., and C.T.M. designed research; A.M.P., M.P., and A.H. performed research; A.M.P., M.P., and C.T.M. analyzed data; A.M.P., M.P., and C.T.M. wrote the paper.

This work was supported in part by National Institutes of Health (NIH) Grants 1R01AG036871 and 5R01EY010804 (C.T.M.); and NIH Grants 5T32NS007492 and 5T32NS007459, the American Heart Association, predoctral grant 11Pre7610007, and the Lois Pope LIFE Fellowship (A.M.P.).

${ }^{*}$ A.M.P. and M.P. contributed equally to this work.

The authors declare no competing financial interests.

Correspondence should be addressed to Carlos T. Moraes, 1420 NW 9th Avenue, Room 229, Miami, FL 33136. E-mail: cmoraes@med.miami.edu.

DOI:10.1523/JNEUROSCI.4871-11.2011

Copyright $\odot 2011$ the authors $\quad 0270-6474 / 11 / 3117649-10 \$ 15.00 / 0$
}

tem brains have decreased mitochondrial complex I activity within the affected SN (Schapira et al., 1990a,b). MPTP and its metabolized byproduct MPP +, first found to cause atypical parkinsonism, work through selective uptake mechanisms of dopaminergic neurons inhibiting complex I (Vyas et al., 1986). Also, systemic exposure to complex I-inhibiting pesticides causes a similar parkinsonian neurodegenerative phenotype (Betarbet et al., 2000; Shimohama et al., 2003). More recently, indicators of a possible role for mitochondria-related energy dysregulation in PD were found during in vivo imaging studies in early- and latestage PD patients; these studies have shown a decrease of energy sources (ATP and phosphocreatine, a rapid mobilizer of ADP to ATP) in patients' midbrain and dorsal striatum (Hattingen et al., 2009).

Other OXPHOS complexes seem to also be affected and contribute to the overall decline in mitochondrial bioenergetics. Notably, SN dopaminergic neurons in both PD and aged individuals have been found to harbor high levels of mitochondrial DNA (mtDNA) deletions (up to 60\% mtDNA deletion load) that were associated with cytochrome $c$ oxidase (complex IV) dysfunction (Bender et al., 2006; Kraytsberg et al., 2006; Reeve et al., 2008). mtDNA is particularly important, as it encodes for subunits that contribute to all the OXPHOS complexes except complex II (Anderson et al., 1981). Thus, it would be reasonable to anticipate that age-related mtDNA mutations would impair global OXPHOS and 
general energy metabolism, either conferring a risk for PD or exacerbating its symptoms.

In this study, we generated a novel mouse model of PD to investigate how mtDNA damage and its consequent OXPHOS defect could affect the physiology of dopaminergic neurons and its effect on behavioral decline. We used a transgenic mouse model expressing PstI endonuclease targeted to the mitochondria, mito-PstI. Mito-PstI causes double-strand breaks in the mtDNA, both depleting the levels of mtDNA and leading to the formation of large deletions. This results in a chronic OXPHOS deficiency by reducing the availability of mtDNA encoded subunits (Srivastava and Moraes, 2005; Fukui and Moraes, 2009). We report here that dopaminergic neuron-specific expression of mito-PstI causes a motor behavioral decline in mice before the loss of SN tyrosine hydroxylase-positive neurons due to neurotransmission defects in the striatal axonal projections.

\section{Materials and Methods}

Animals. The generation of mito-PstI transgenic mice was previously described (Fukui and Moraes, 2009). Briefly, a mammalianized version of the bacterial PstI gene was positioned behind a 5'mitochondrial targeting sequence from human COX VIII gene (cytochrome $c$ oxidase subunit VIII). The intervening sequence 8 (IVS8) was introduced between the tetracycline response element (TRE) promoter sequence and the mito-PstI coding sequence. The transgenic line used in this study is previously described Line 5751 (Fukui and Moraes, 2009). Mice were backcrossed with C57BL/6J mice for colony maintenance. The nuclear background was mostly C57BL/6J (backcrossed 7 generations) for male animals used for behavioral testing. No behavioral differences were found between littermates of the same mito-PstI genotype. Biochemical analyses were performed in male animals that were subjected to additional backcrosses with C57BL/6J (10 generations). These mice of the same background were crossed with transgenic mice harboring one allele for a dopamine transporter-driven mammalianized tetracycline transactivator protein (DAT-tTA) (Cagniard et al., 2006).

All mice procedures were performed according to a protocol approved by the University of Miami Institutional Animal Care and Use Committee. Mice were housed in a virus-antigen-free facility at the University of Miami Division of Veterinary Resources in a $12 \mathrm{~h}$ light/dark cycle at room temperature and fed ad libitum with a standard rodent diet.

Pole test. Pole test for motor coordination/nigrostriatal dysfunction of mice was previously described (Matsuura et al., 1997). Animals were hung upright on a vertical ( $8 \mathrm{~mm}$ diameter; $55 \mathrm{~mm}$ length) pole and were given $3 \mathrm{~min}$ to change orientation to descend. Animals were given three trials with an average taken of the latency to descent to the base. Failure to descend or fall from the pole was given a maximum time of $3 \mathrm{~min}$.

RotaRod. Motor coordination was evaluated with a RotaRod (IITC Life Sciences) designed for mice. Animals were tested on a monthly basis with four runs on a given day with two runs for practice. The final two runs were recorded and combined to find the average latency to fall. A resting period of $45 \mathrm{~min}$ between each run was given. Animals were required to position limbs to stay on a rotating rod accelerating from 6 to $20 \mathrm{rpm}$ over a $300 \mathrm{~s}$ time period. Mice that completed the task received a final latency time of $300 \mathrm{~s}$.

Activity monitoring. Spontaneous self-initiated movement was recorded using an activity cage setup (Columbus Instruments) designed for mice. Animals were housed in a novel cage environment separately 30 min before their dark cycle and monitored for a 12 or $24 \mathrm{~h}$ period undisturbed. Ambulatory movement was counted by the number of infrared beam breaks that occurred inside of the cage.

Pharmacological treatment. Mice were treated with L-3,4dihydroxyphenylalanine (L-DOPA) (200 mg/kg) and benserazide (25 $\mathrm{mg} / \mathrm{kg}$ ) (Sigma) administrated via intraperitoneal injection $1 \mathrm{~h}$ before behavioral testing.

Statistical analysis for behavior. PD-mito-PstI mice (PstI+/DATtTA,$+ n=9$ ) and their control counterparts (mito-PstI-/DAT-tTA + , $n=11$ ) were weighed monthly and subjected to all behavioral character- izations as described. Data were analyzed using a two-tailed, unpaired Student's $t$ test. Differences were considered significant when $p<0.05$.

Immunohistochemistry. Anesthetized mice were killed using cervical dislocation. Brains were quickly isolated and submerged in $4 \%$ paraformaldehyde at $4^{\circ} \mathrm{C}$ overnight and then cryoprotected by increasing concentrations of sucrose solutions $(10 \%, 20 \%, 30 \%)$ prepared in PBS. The cryoprotected brains were positioned in a plastic block (Polysciences), submerged in OCT Compound solution (TissueTek) diluted with an equal volume of $25 \%$ sucrose, and quickly frozen by submerging the plastic block into 2-methylbutane cooled in liquid nitrogen. Twenty- or $30-\mu \mathrm{m}$-thick sections were cut on a cryostat (Leica) and used or stored at $-80^{\circ} \mathrm{C}$.

For neuron counting, $30 \mu \mathrm{m}$ sections were thawed to room temperature, washed in PBS, and permeabilized with $0.4 \%$ Triton X-100. Slides were then blocked for $1 \mathrm{~h}$ at room temperature with normal goat serum (KPL) and incubated with primary antibody anti-tyrosine hydroxylase (Sigma) at 1:500. Secondary mouse conjugated HRP antibody (KPL) was used for $1 \mathrm{~h}$ at room temperature. Slides were subsequently detected with the addition of streptavidin-peroxidase (KPL) for $30 \mathrm{~min}$ and visualized with $0.05 \%$ 3,3'-diaminobenzidine (DAB) for $7 \mathrm{~min}$. Slides were dehydrated with increasing concentrations of ethanol and cleared with xylene before mounting with Sub-X mounting medium (Surgipath). Images were captured with an OLYMPUS BX51 microscope. For fluorescent immunohistochemistry images, 20 or $30 \mu \mathrm{m}$ sections were thawed at room temperature, washed in PBS, and permeabilized with $0.4 \%$ Triton $\mathrm{X}-100$. Slides were blocked with $2 \%$ FGS and $1 \%$ BSA for $1 \mathrm{~h}$ at room temperature and incubated with primary antibody anti- $\alpha$-synuclein 1:500 (Cell Signaling Technology), anti-ubiquitin 1:500 (Cell Signaling Technology), anti-tyrosine hydroxylase (Sigma) at 1:500, and anti-PstI at 1:500 (Srivastava and Moraes, 2001) overnight at $4^{\circ} \mathrm{C}$. Alexa 488 goat anti-mouse or Alexa 594 goat anti-rabbit-conjugated secondary antibodies (Invitrogen) were used for $1 \mathrm{~h}$ at room temperature in the dark. Slides were then mounted with fluorescent mounting media or mounting media with DAPI (Vector Laboratories). Images were captured with a LSM710 confocal microscope (Zeiss).

Western blotting. Protein extracts were prepared from isolated striatum samples homogenized with a hand-held rotor (VWR) in PBS containing a protease inhibitor cocktail (Roche). Samples were then snap frozen in liquid nitrogen and stored at $-80^{\circ} \mathrm{C}$ until use. Upon use, SDS was added to the homogenate at the final concentration of $4 \%$. Homogenates were then sonicated and centrifuged at $14,000 \times g$, and the supernatant was collected for analysis. Proteins were quantified using Dc Protein Assay kit (Bio-Rad) using Lowry HS methodology. Proteins were run on either a $12 \%$ SDS-acrylamide gel or 4-20\% SDS-polyacrylamide gradient gel (Bio-Rad) depending on the target's predicted molecular weight. The gel was blotted on polyvinylidene fluoride (PVDF) (BioRad) or nitrocellulose (Bio-Rad) membrane.

Membranes were blocked in 1:1 Odyssey blocking solution (LI-COR Biosciences) for $1 \mathrm{~h}$ at room temperature. Primary antibodies used were mouse anti- $\alpha$-tubulin 1: 2000 (Sigma), mouse anti-tyrosine hydroxylase 1:1000 (Sigma), goat anti-myelin basic protein 1:1000 (Millipore), antidopamine transporter 1:1000 (Sigma), and rabbit anti-actin 1:5000 (Sigma). Primary antibodies were incubated overnight at $4^{\circ} \mathrm{C}$. Secondary antibodies used were infrared-conjugated antibodies anti-rabbit-700/ anti-mouse- 800 (Rockland) at 1:3000 to 1:5000 concentrations. Secondary antibodies were incubated for $1 \mathrm{~h}$ at room temperature. Blots with infrared secondary antibodies were visualized with Odyssey Infrared Imaging System (LI-COR Biosciences). Optical density measurements were taken by default software supplied by LI-COR on blots.

For Western blot exposure with x-ray, secondary anti-chickenHRP or anti-mouse (Cell Signaling Technology) was used at 1:1000 or 1:5000 dilution, respectively. Incubation occurred for $1 \mathrm{~h}$ at room temperature. SuperSignal Chemiluminescent HRP substrate (Pierce) was used to visualize the bands with Hyclone autoradiography film (Denville Scientific).

Stereological neuron counting. Stereology workstation program (StereoInvestigator; MicroBrightField) was used to quantify $\mathrm{TH}+$ neurons. Immunohistochemical identification of $\mathrm{TH}+$ neurons is described above. Dopaminergic neurons were counted in randomly selected serial 
A

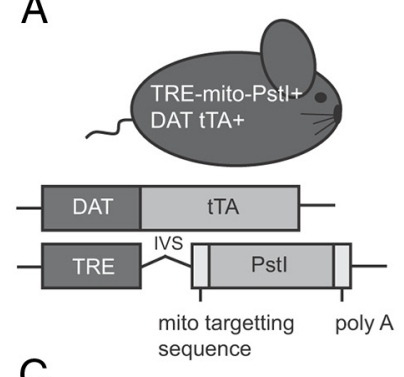

C
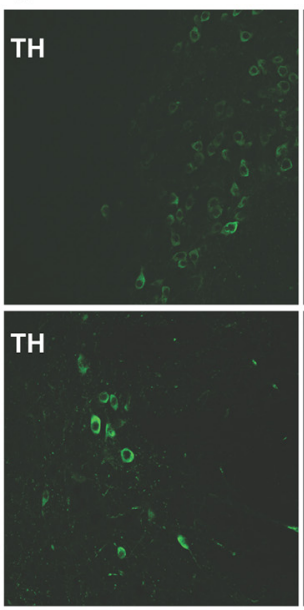

Pstl
B

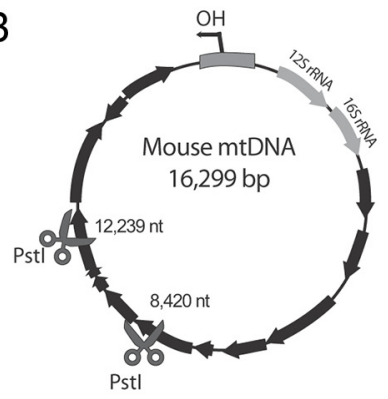

Pstl

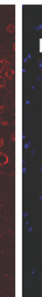

\section{merge}
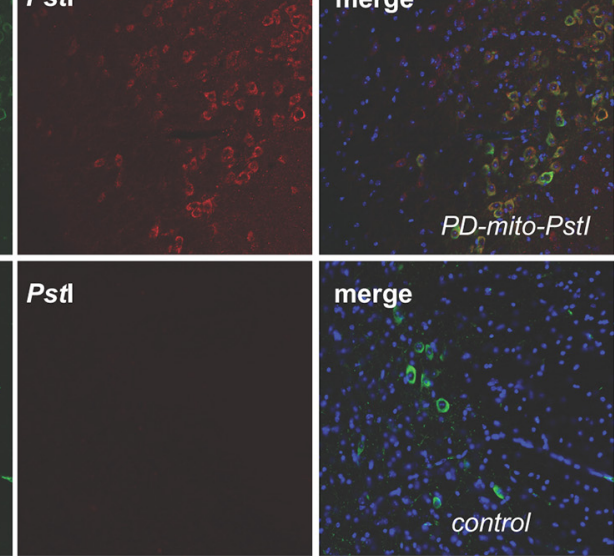

merge

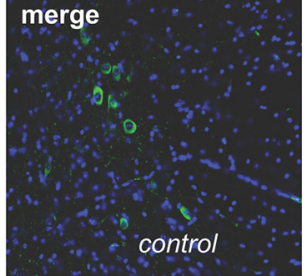

$\mathrm{D}$
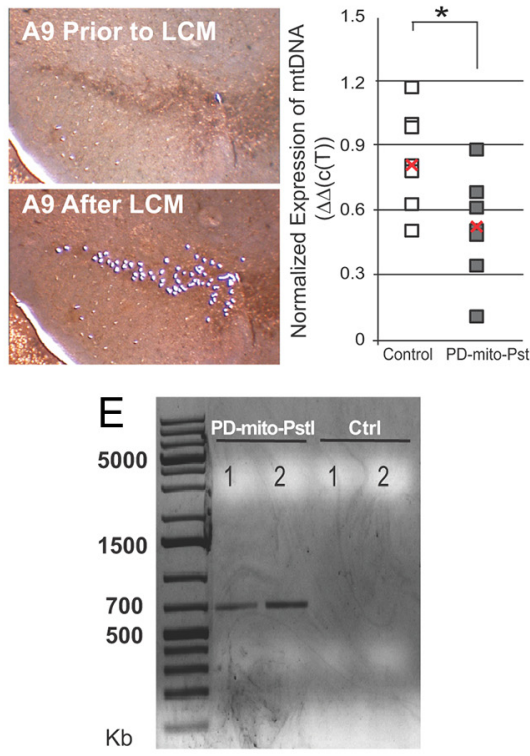

Figure 1. Mito-Pstl expression in PD-mito-Pstl mouse dopaminergic neurons and alterations in nigral-striatal mtDNA. A, Schematic representation of the experimental PD-mito-Pstl mouse and the transgenic constructs it harbors. $\boldsymbol{B}$, mtDNA map showing the targeted sites of Pstl at nt 8420 and 12,239 (scissors). Black arrows denote protein-coding genes. $\boldsymbol{C}$, Immunohistochemical images of colocalization of mito-Pstl protein and TH-immunopositive cells in the SN region of 4-month-old PD-mito-Pstl animals (upper panels) and in age-matched negative controls (lower panels). $\boldsymbol{D}$, Representative images of $\mathrm{A} 9 \mathrm{TH}+$ cells collected by laser capture microscopy (left, 10× magnification) and quantification of mtDNA levels of TH + cells from individual mice by real-time PCR (right). Each square represents one mouse. The red " $x$ " represents the mean value. Results were normalized to the internal control gene actin $\left(n=7 /\right.$ group, $\left.{ }^{*} p<0.05\right)$. $E$, Detection of mtDNA rearrangements. PCR products from 6-week-old PD-mito-Pstl mice striatal DNA using primers 8300F and 12812B, which flank the Pstl endonuclease sites, confirmed the presence of recombined molecules resulting from the double strand breakage.

sections through the midbrain with $10-12$ sections counted per individual animal. TH + cells numbers were determined on $30 \mu \mathrm{m}$ immunohistochemical stained sections. According to the program, we set a guard zone of $3 \mu \mathrm{m}$ from the top and from the bottom of each section that was not considered in the counting. As the estimated actual section thickness after processing was $\sim 20 \mu \mathrm{m}$, the actual dissection height where counting occurred was $14 \mu \mathrm{m}$. The A9 area was outlined using the $10 \times$ objective and neurons were counted at $60 \times$ magnification with oil on an Olympus BX51 microscope. A scan grid size was determined to have at least 10 grid sites per section. The estimated numbers of dopaminergic neurons were calculated by counting $\mathrm{TH}+$ cells present in the unbiased virtual inclusion counting frames. We accepted a coefficient of error (Gunders) of $<0.15$.

Dopamine and metabolite quantification. Dopamine and metabolite quantification measurements were performed by the Vanderbilt University CMN/KC Neurochemistry Core Lab. Freshly isolated striatum was harvested and quickly frozen in liquid nitrogen from mice killed as previously described.

Laser capture microdissection. Thirty micrometer sections were immunostained for TH as described above using DAB as detection substrate. Slides were dehydrated in gradient alcohol washes, cleared with xylenes, and air dried. Sections were placed in a chamber with desiccant chips (1 g/each) overnight to facilitate drying of the sections. TH-positive neurons in the $\mathrm{SN}$ region were excised from $\sim 10$ slides per animal using LMD LeicaLaser Microdissection microscope (Leica).

DNA was extracted using the QIAmp DNA Micro Kit (Qiagen) and was subsequently used for quantitative real-time PCR to measure mtDNA levels. We designed a couple of primers (see below) to amplify mtDNA in a region not containing a PstI site to measure both undeleted and potentially deleted or recombined molecules. To determine relative quantity of mtDNA in each sample, we used the comparative Ct method (Schmittgen and Livak, 2008), normalizing the amplification with primers that amplify a genomic DNA region. Relative quantity was corrected for relative PCR amplification efficiency using Biorad CFX Manager Software.

Primers for mtDNA were as follows: ND1 $3281 \mathrm{~F}$, CAGCCTGACCCATAGCCATA; ND1 3364 B, ATTCTCCTTCTGTCAGGTCGAA. Primers for genomic DNA were as follows: $\beta$-actin F, GCGCAAGTACTCTGTGTGGA; $\beta$-actin B, CATCGTACTCCTGCTTGCTG. Targets were amplified using Maxima SYBR Green/ROX qPCR Master Mix (Fermentas) using CFX96 Realtime PCR system (Bio-Rad) under the suggested PCR conditions from the manufacturer.

$m t D N A$ deletion detection. DNA was isolated from the striatal homogenates using phenol:chloroform extraction. DNA was used in a PCR to detect potential recombination events that involve mtDNA deletions at the PstI restriction endonuclease sites. The primers set used against mtDNA flanked the two PstI sites: at position 8300 (TTGCCCACTTCCTTCCACAAG) and at position 12,812 (TCGGATGTCTTGTTCGTCTG). This PCR was expected to amplify $4.5 \mathrm{kbp}$ bands in control samples in which there was no recombination of mtDNA. However, if there was recombination in mtDNA somewhere between nt 8300 and 12,812 , this primer set would preferentially amplify a shorter breakpoint region.

PCR conditions were as follows: 3 min at $94^{\circ} \mathrm{C} ; 30$ cycles of $94^{\circ} \mathrm{C}$ for $30 \mathrm{~s}, 60^{\circ} \mathrm{C}$ for $1 \mathrm{~min}$, and $72^{\circ} \mathrm{C}$ for $75 \mathrm{~s}$; and a final extension time of 10 min of $72^{\circ} \mathrm{C}$. Two hundred fifty nanograms of DNA were used as template with LA Taq polymerase (TAKARA). PCR products were sequenced to confirm recombination events at PstI restriction endonuclease sites. 
A

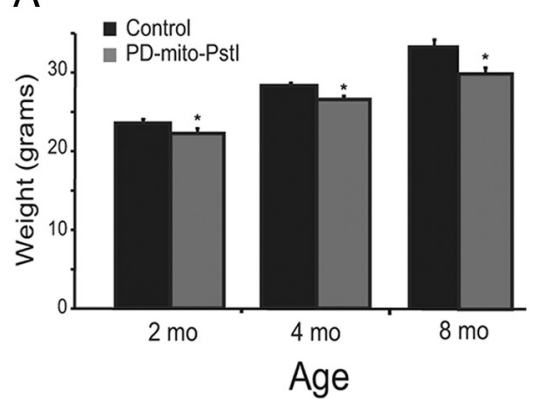

D

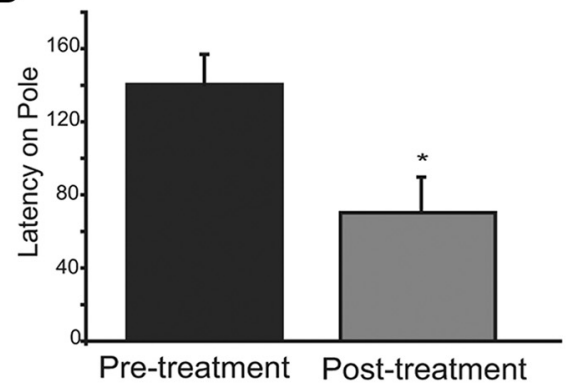

$B$

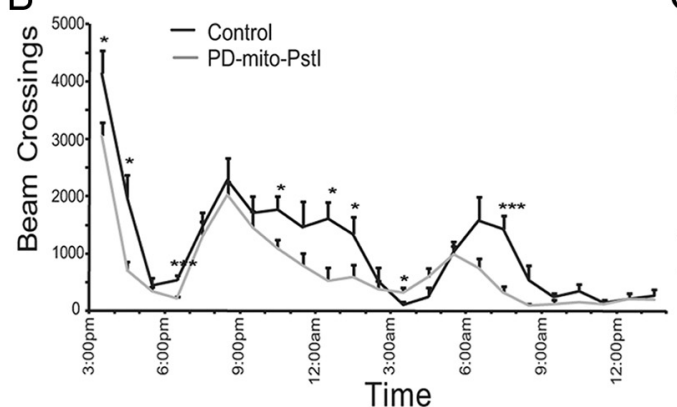

E

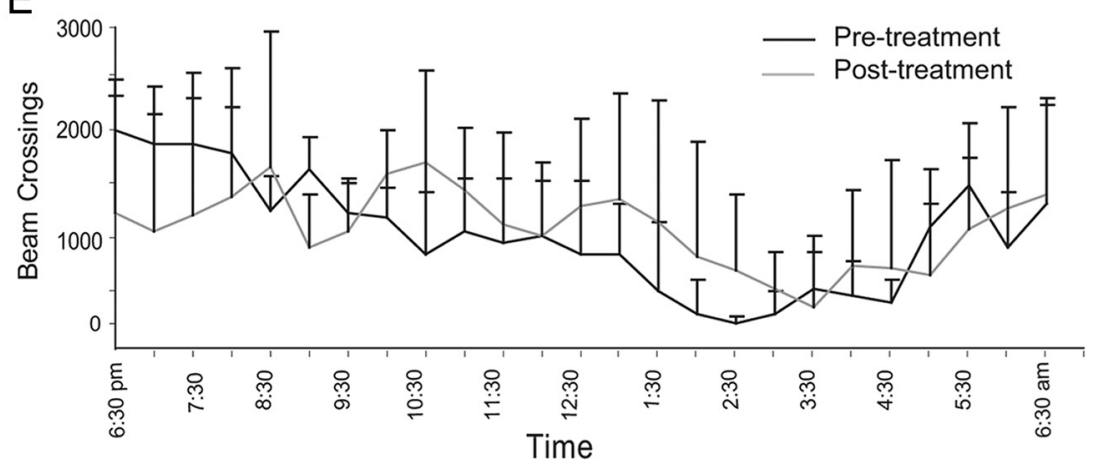

Figure 2. PD-mito-Pstl mice display abnormal motor behaviors. A, Body weight measurement of 2-, 4-, and 8-month-old animals: PD-mito-Pstl showed a small but significant reduction in weight compared to age-matched controls. $\boldsymbol{B}$, Activity test on 2-month-old animals: PD-mito-Pstl mice have less exploratory behavior and spontaneous activity compared to controls. $\boldsymbol{C}$, Pole test on 2- and 4-month-old animals: 4-month-old PD-mito-Pstl mice displayed increased latencies times when required to descend a pole, as compared to controls. $\boldsymbol{D}, \boldsymbol{E}$, Pole test and activity cage on 4-month-old PD-mito-Pstl animals, before (pre-treatment) and after (post-treatment) intraperitoneal administration of L-D0PA (200 mg/kg) and carbidopa (25 mg/kg): performance latency was

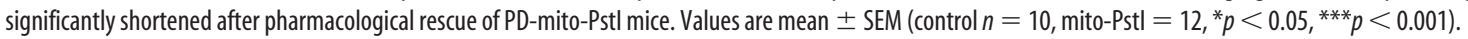

\section{Results}

\section{Characterization of dopaminergic neuronal expression of} mito-PstI in transgenic mice

In mito-PstI mice, the expression of mito-PstI is regulated by a minimal promoter containing a TRE. We mated mito-PstI transgenic mice with DAT-tTA mice that express a mammalianized tetracycline transactivator (tTA) protein under the control of the endogenous dopamine transporter promoter (gene knock-in in the DAT locus). Subsequent matings produced animals that were positive for both alleles (mito-PstI+/DAT-tTA+) (Fig. 1A). For simplicity, we refer to these double transgenic mice as "PD-mitoPstI" mice. In this tet-off system, in the absence of doxycycline, tTA protein expressed under the DAT promoter binds to TRE promoter and induces the expression of mito-PstI. Siblings that were positive only for DAT-tTA allele (mito-PstI-/DAT-tTA+) do not express mito-PstI protein and therefore were used as controls. Male mice (tet-off) were continuously induced from birth by keeping them on a standard rodent diet. Mito-PstI cleaves the mouse mtDNA at nucleotide positions 8420 and 12,238 (Fig. $1 B$ ), generating both deletions and a severe depletion (Srivastava and Moraes, 2005; Fukui and Moraes, 2009; Pickrell et al., 2011).

To detect the expression of mito-PstI in dopaminergic neurons, we performed double immunohistochemistry with antimito-PstI and anti-tyrosine hydroxylase (TH), the rate-limiting enzyme for catecholamine biosynthesis. In PD-mito-PstI animals' midbrain sections, we observed a mito-PstI/TH colocalization in SN (Fig. 1C). Staining of the mito-PstI protein was absent in controls (Fig. 1C).

\section{Confirmation of the functional effect of mito-PstI in vivo}

To test the functionality of mito-PstI enzyme in our model, we isolated TH-immunopositive cell bodies in PD-mito-PstI and controls at 4 months of age using laser capture microdissection. Using quantitative PCR (see Materials and Methods), we observed mtDNA depletion in some PD-mito-PstI mice at this time point (Fig. 1D). We also performed a PCR on DNA samples from 6-week-old PD-mito-PstI mice striata, using primers and extension times that would amplify recombined mtDNA if present (see Materials and Methods). We detected unique PCR products that were absent in age-matched controls (Fig. $1 E$ ). Cloning and sequencing these unique bands confirmed the presence of mtDNA recombination events close to the two PstI cleavage sites at positions 8420 and 12,239 (i.e., breakpoints at: $8423-12,825,8401-$ $12,825$, and $8391-12,825)$. We also detected mtDNA deletions in 4-month-old PD-mito-PstI striata (data not shown).

Although there are many different cell types in the brain that can mask the effects of mito-PstI on a smaller dopaminergic neuronal population, we were able to document mtDNA damage caused by mito-PstI (the presence of mtDNA deletions and reduction in mtDNA levels) as previously observed when mito-PstI was expressed in other CNS regions (Fukui and Moraes, 2009; Pickrell et al., 2011).

\section{Motor-related behavioral abnormalities in \\ PD-mito-PstI mice}

We followed the general behavior and physical condition of PDmito-PstI mice and age-matched control animals at 2, 4, 8 and $>12$ months of age (mice between 12 and 14 months of age). PD-mito-PstI animals appeared normal at birth and at expected Mendelian ratios. There were no overt health problems up to the time they were killed at 18 months $(n=15)$. However, we noticed the first phenotype at 2 months of age where PD-mito-PstI animals gained weight at a slower rate than control counterparts. A similar pattern was observed at different ages (Fig. 2A). 

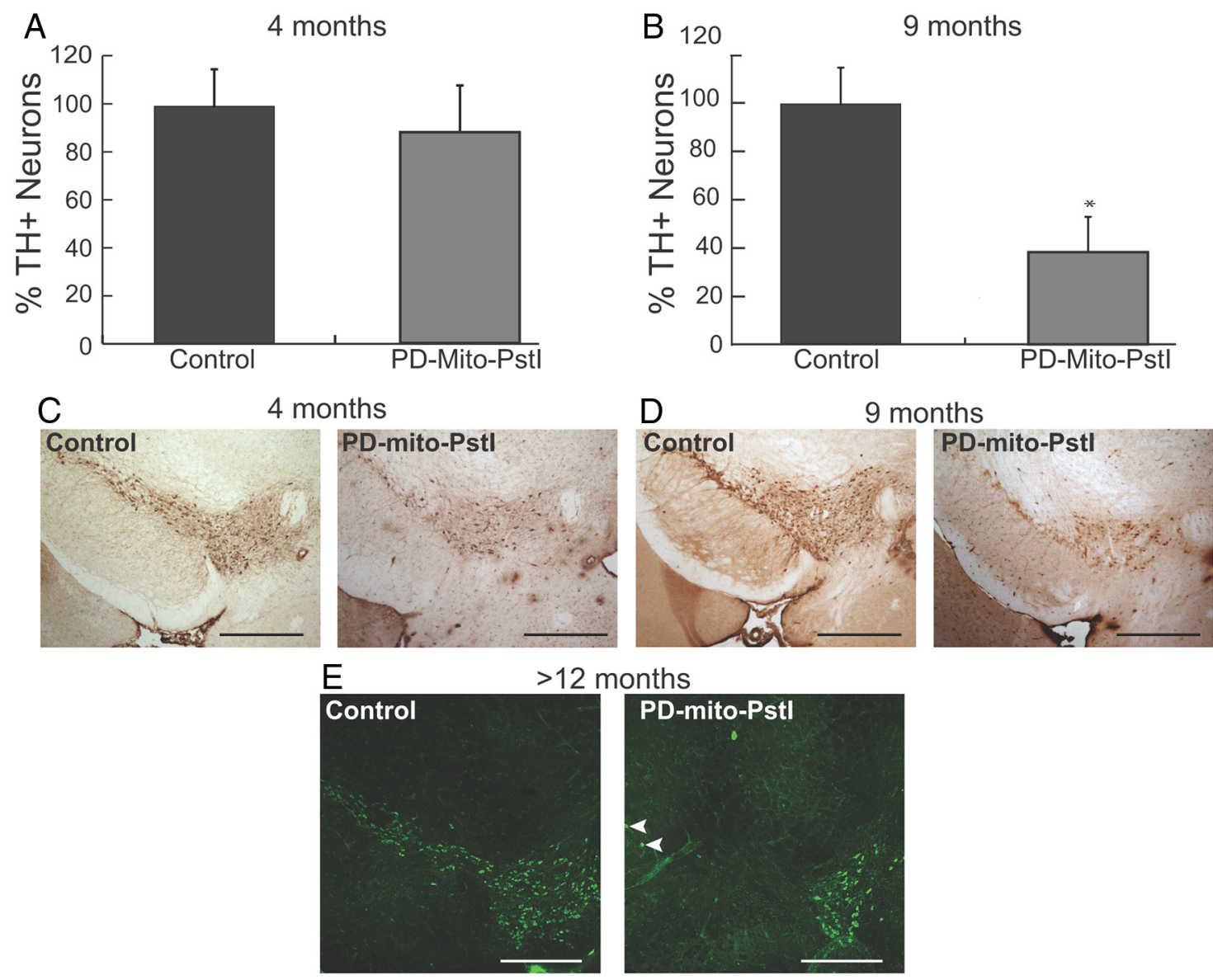

Figure 3. Progressive loss of A9 tyrosine hydroxylase-positive neurons in the PD-mito-Pstl mice. A, Quantification of A9 TH + neuronal populations in 4-month-old animals: PD-mito-Pstl mice showed no significant differences in TH + neurons percentage as compared to controls $B$, Quantification of A9 TH + neuronal populations in 9-month-old animals: PD-mito-Pstl mice showed an $\sim 40 \%$ reduction in the amount of $\mathrm{TH}+$ neurons compared to controls. $C, D$, Representative immunohistochemical identification of $\mathrm{TH}+$ neurons sections used for quantification at 4 months ( $C$ ) and 9 months (D) of age. $\boldsymbol{E}$, Representative immunohistochemical staining of TH in midbrain of $>12$-month-old animals: PD-mito-Pstl showed the absence of SN region and a milder degeneration of dopaminergic neurons in VTA ( $n=4$ /group). White arrowheads depict rare TH + neuronal bodies in A9 of $>12$-month-old animals. Values are mean \pm SEM (control $n=4-5$, PD-mito-Pstl $=$ $\left.4-5,{ }^{*} p<0.05\right)$. Scale bars, $5 \mu \mathrm{m}$.

As mito-PstI was being targeted specifically to dopaminergic neurons, we analyzed motor coordination and normal locomotion deficits. When measuring spontaneous self-initiated activity at 2 months of age, we observed a significant decrease in the exploration time in the activity cage with this reduction lasting the duration of the dark cycle for PD-mito-PstI mice (Fig. 2B). We used a pole test behavioral assay (see Materials and Methods) to assess the ability of the mouse to coordinate limbs to descend a pole from a fixed height. At 2 months of age, there was no significant difference between the latencies of control and PD-mitoPstI animals. However, at 4 months of age, PD-mito-PstI mice showed significant increases in their latency time as compared to controls, indicative of poor motor coordination (Fig. 2C).

A common therapy to improve symptoms of $\mathrm{PD}$ is the administration of L-DOPA and carbidopa. L-DOPA drug facilitates the production of dopamine in surviving dopaminergic neurons alleviating symptoms of the disease, whereas carbidopa is used to antagonize the peripheral conversion of L-DOPA to dopamine. We treated 4-month-old PD-mito-PstI mice with L-DOPA $(200 \mathrm{mg} / \mathrm{kg})$ and carbidopa $(25 \mathrm{mg} / \mathrm{kg})$ (i.p.) and performed pole test and activity testing before the injection and after $1 \mathrm{~h}$ after the pharmacological rescue. After treatment, PD-mito-PstI performed significantly better on the pole test and performed no worse in the activity cage as com- pared to baseline behavior (Fig. $2 D, E$ ). The responsiveness to L-DOPA treatment on the pole test led us to confirm that at the 4 month time point, $\mathrm{PD}$-mito-PstI already have a nigrostriatal defect caused by dopamine dysfunction.

We performed another test of motor coordination using the RotaRod at 2, 5, and 8 months with no significant difference between the latencies of PD-mito-PstI mice and controls (data not shown). This was not surprising, as it has been shown (Meredith and Kang, 2006) that tests of walking or climbing are better able to detect parkinsonian motor impairments, while measurements such as time spent on the Rotarod or general activity monitoring do not directly assess basal ganglia deficits. Therefore, this model of mitochondrial dysfunction in dopaminergic neurons caused a relatively mild PD phenotype.

\section{Progressive loss of SN tyrosine hydroxylase-positive neurons populations}

We then analyzed the dopaminergic neuronal populations in the $\mathrm{SN}$ of PD-mito-PstI mice. At 4 months of age, when motor coordination became impaired, the number of $\mathrm{SN} \mathrm{TH}+$ neurons was not significantly different between PD-mito-PstI mice and agematched controls (Fig. $3 A, C$ ). When we quantified this same population at a later time point ( 9 months of age), we found a reduction of approximate $60 \%$ in $\mathrm{TH}+$ cell bodies in the $\mathrm{SN}$ 
A

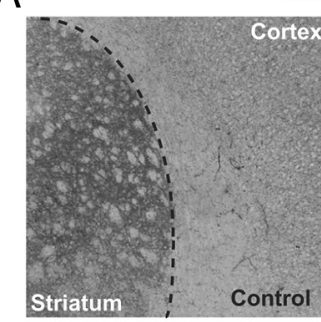

4 months

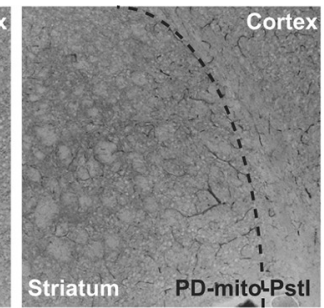

C
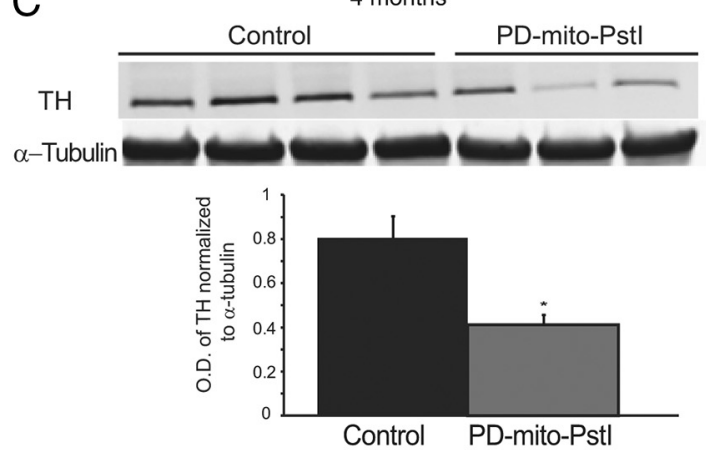

E

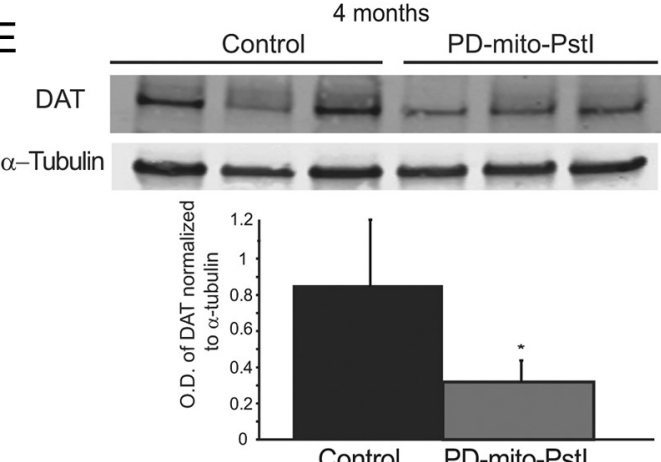

B
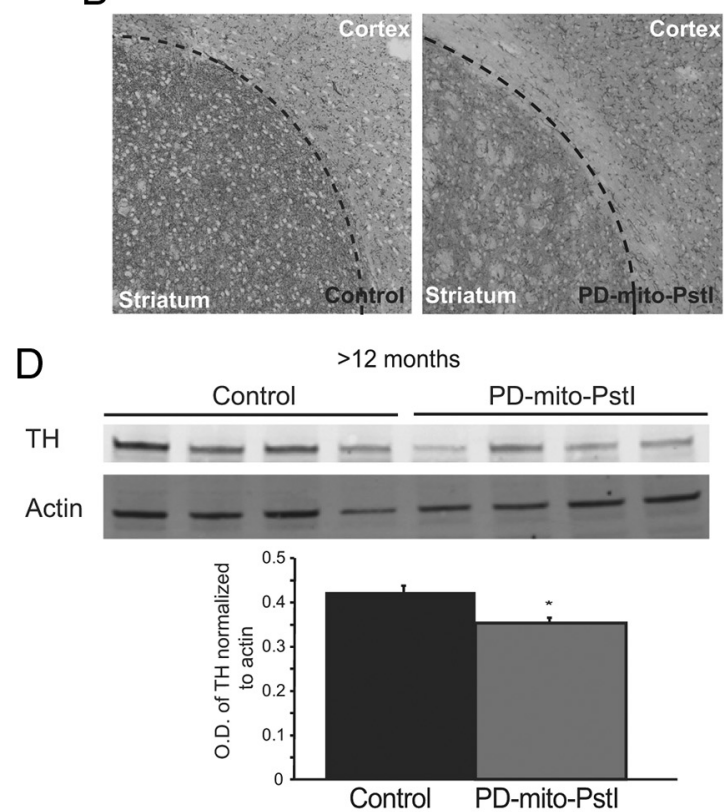

F

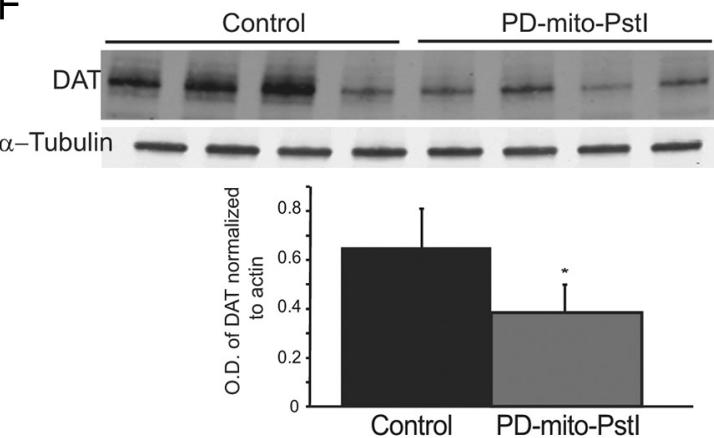

Figure 4. TH and DAT are reduced in the striatum at 4 and at 12 months of age in PD-mito-Pstl mice. $A, B$, Representative grayscale images of fluorescent immunostaining of TH on striatal slides of 4- ( $\boldsymbol{A})$ and $>12-(\boldsymbol{B})$ month-old animals. $\boldsymbol{C}, \boldsymbol{D}$, Western blotting probing for TH in striatal homogenates of 4- ( $\boldsymbol{C}$ and $>12-(\boldsymbol{D})$ month-old PD-mito-Pstl mice and controls and optical density (0.D.) quantification of TH normalized to $\alpha$-tubulin: both techniques show a decrease in TH detection in PD-mito-Pstl mice. $\boldsymbol{E}$, $\boldsymbol{F}$, Western blotting probing for DAT in striatal homogenates of 4- $(\boldsymbol{E})$ and $>12$ - $(\boldsymbol{F})$ month-old PD-mito-Pstl mice and controls and 0.D. quantification of DAT normalized to $\alpha$-tubulin showing a decrease in DAT steady-state protein levels in PD-mito-Pstl mice compared to controls. Values are mean \pm SEM $\left({ }^{*} p<0.05\right)$.

region of PD-mito-PstI animals (Fig. $3 B, D$ ). At 12 months of age, there were very few TH-positive neurons left in the SN of PDmito-PstI mice, although they were abundant in the VTA (Fig. $3 E$ ). The numbers of $\mathrm{TH}+$ neurons at this time point were too small for quantification with the Neurolucida methodology. These results suggest that, in our model, the behavioral phenotypes observed early on occur before the massive loss of SN tyrosine hydroxylase-positive neuronal cell bodies.

\section{Striatum alterations in the PD-mito-PstI model}

Because we noted no significant SN cell body loss at 4 months of age, when the locomotive deficits appear, we analyzed changes in the striatum that may have contributed to the phenotype in our model. We performed an immunohistochemical staining in striatum sections of 4-month-old PD-mito-PstI, and observed a decrease in the TH signal as compared to controls (Fig. 4A). We confirmed this result by Western blot of striatal homogenates from PD-mito-PstI mice as compared to controls (Fig. 4B). This significant decrease in $\mathrm{TH}$ was also observed in striatal homogenates from older PD-mito-PstI mice ( $>12$ months of age) (Fig. 4C,D).

To investigate whether this reduction in $\mathrm{TH}$ in the striatum was due to axonal degeneration of the projecting fibers from the SN cell bodies, we analyzed the steady-state levels of DAT (a presynaptic membrane protein) in striatal homogenates. At 4 months of age, we detected a significant reduction in DAT steadystate levels in the striatum of PD-mito-PstI mice (Fig. $4 E$ ), suggesting that dopaminergic presynaptic terminals of the striatum are already reduced at 4 months, before cell body loss. This decrease persisted and increased in PD-mito-PstI mice at $>12$ months of age compared to controls (Fig. $4 F$ ).

\section{Dopamine neurotransmitter and degradation product defects} in the striatum

As PD motor symptoms are due to the depletion of dopamine in the striatum, we quantified the amount of dopamine and its downstream metabolites in freshly isolated striatum samples, starting from 4 months of age, when motor symptoms appeared. There was 

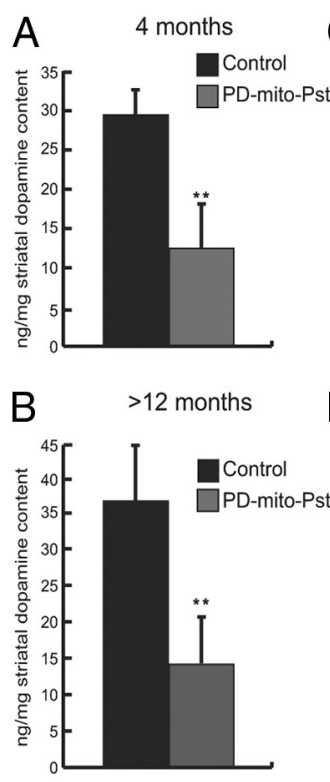

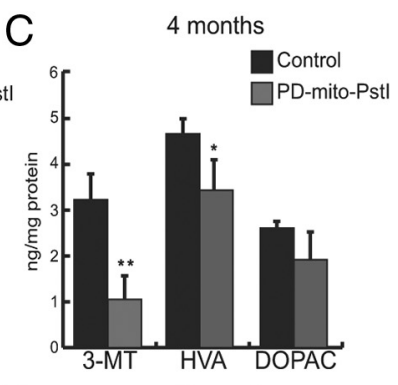

D

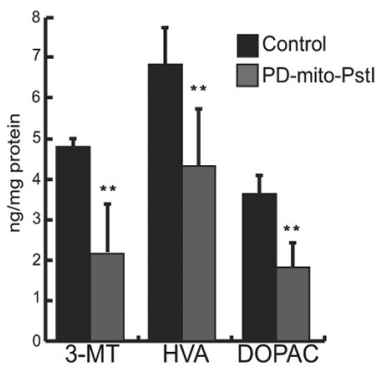

$E$
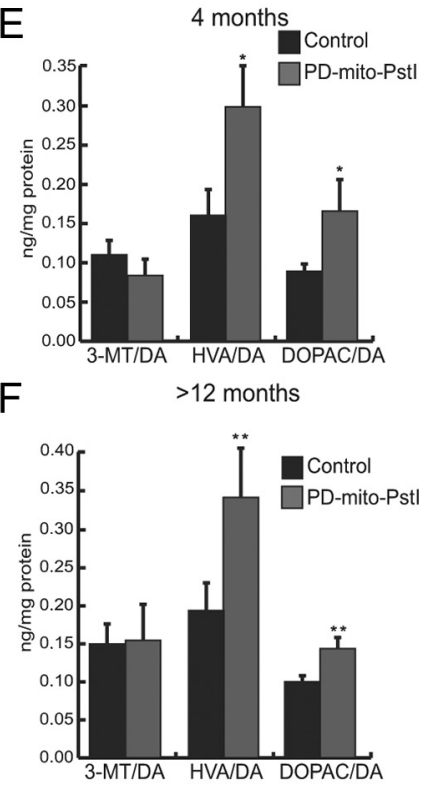

Figure 5. Dopamine and neurotransmitter degradation products defects in PD-mito-Pstl mice. $A, B$, Dopamine quantification from striatal homogenates of $4-(\boldsymbol{A})$ and $>12-(\boldsymbol{B})$ month-old PD-mito-Pstl and control animals (values normalized to milligrams of protein, $n=4$ /group). C, D, Quantification of 3-MT, HVA and DOPAC from striatal homogenates of 4- (C) and $>12$ - (D) month-old animals: 4-month-old PD-mito-Pstl show a reduction of 3-MT and HVA but not a significant change in DOPAC compared to controls (values normalized to milligrams of protein, $n=4$ /group). More-than-12-month-old PD-mito-Pstl also showed a reduction of DOPAC compared to controls (values normalized to milligrams of protein, $n=6 /$ group). $E, F$, Ratio of dopamine metabolites to dopamine content in 4- $(\boldsymbol{E})$ and $>12-(\boldsymbol{F})$ month-old animals: PD-mito-Pstl mice show a relative increase for HVA and DOPAC ( $n=4-6 /$ group). Values are mean $\pm \operatorname{SEM}\left({ }^{*} p<0.05,{ }^{* *} p<0.01\right)$.

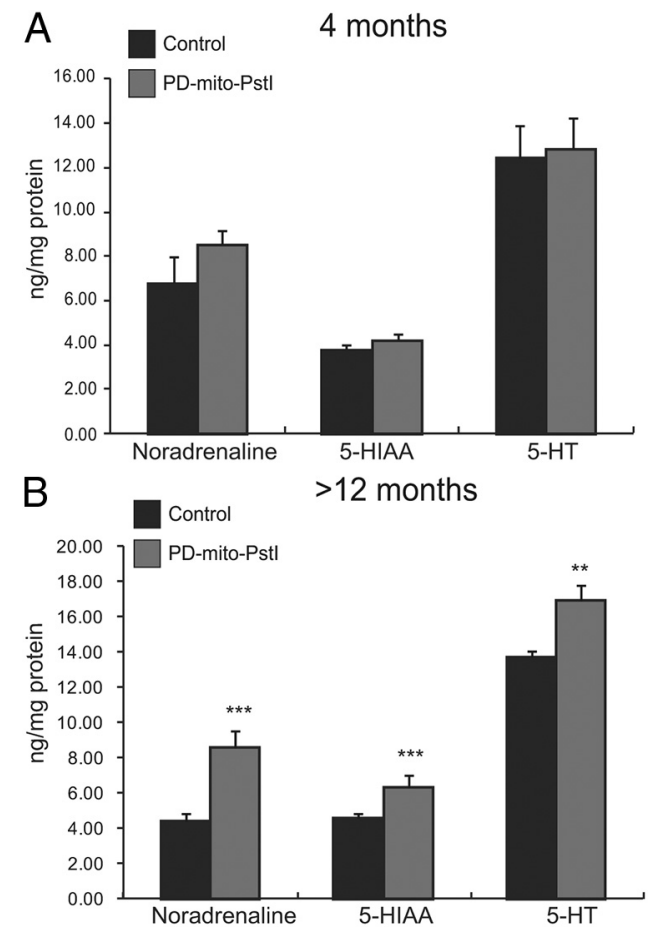

Figure 6. Noradrenaline and serotonin increase in PD-mito-Pstl mice at $>12$ months of age. $A, B$, Quantification of NE, 5-HIAA, and 5-HT from striatal homogenates of 4- $(\boldsymbol{A})$ and $>12-(\boldsymbol{B})$ month-old animals: PD-mito-Pstl striatal show a significant increase in NE, 5-HIAA, and 5-HT at $>12$ months of age compared to controls (values normalized to milligrams of protein, $n=$ $4-6 /$ group $)$. Values are mean $\pm \operatorname{SEM}\left({ }^{* *} p<0.01,{ }^{* *} p<0.001\right)$. a significant reduction in the overall amount of dopamine in PD-mito-PstI mice at both 4 and $>12$ months of age compared to their age-matched controls (Fig. 5A,B). We measured the absolute quantity of the three downstream metabolites of dopamine degradation, 3-methoxytyramine (3-MT), homovanillic acid (HVA), and 3,4-dihydroxyphenyl acetic acid (DOPAC). We observed a significant decrease in the HVA and 3 -MT at 4 months of age in PD-mito-PstI mice as compared to controls (Fig. 5C). The same metabolites, as well as DOPAC, were significantly reduced in PD-mito-PstI mice at $>12$ months (Fig. $5 D$ ). When normalizing the metabolites to the amount of available dopamine, there was a significant increase in the amount of HVA and DOPA in PD-mito-PstI striatal samples as compared to controls for both age groups (Fig. $5 E, F)$. Therefore, this bioenergetically impaired model showed striatal altered dopamine metabolism before $\mathrm{SN}$ neuronal body loss.

\section{Increases in other neurotransmitters after the loss of tyrosine hydroxylase-positive neurons}

We next examined other monoamine neurotransmitters and metabolites that were dependent [noradrenaline (NE)] and independent [serotonin (5-HT)] of tyrosine hydroxylase activity; we also measured the levels of se-
rotonin's degradation metabolite 5-hydroxyindoleacetic acid (5HIAA). We detected no significant differences between controls and PD-mito-PstI striatal homogenates in NE, 5-HT, or 5-HIAA at the 4 month time point (Fig. $6 \mathrm{~A}$ ). Surprisingly, PD-mito-PstI mice over 12 months of age showed significant increases in all three monoamine products as compared to age-matched littermates (Fig. 6B). Because Mito-PstI is only being targeted in dopaminergic neurons, these changes in other monoamines and serotonin are likely compensatory responses.

\section{Discussion}

A novel mouse model of PD

We report a novel mouse model of PD, based on a chronic damage of mtDNA in dopaminergic neurons that recapitulates some of the major phenotypes and biochemical changes that occur during the neurodegenerative processes of PD.

In Figure 7, we have summarized the temporal changes in phenotype, patterns of SN neurodegeneration, striatal pathophysiology, and mtDNA changes of our model. At birth, there was no difference between control and PD-mito-PstI animals. We could detect mtDNA deletions early on (6 weeks) and at 4 months in the striatum as well as an mtDNA depletion in the SN at 4 months. These observations showed that the mito-PstI was active throughout the life of the animals, leading to a progressive bioenergetics crisis in the DAT-expressing neurons. The first behavioral phenotypes arise at 2 months of age with a decrease in spontaneous activity in a novel environment and slight weight differences as compared to controls. At 4 months, PD-mito-PstI animals begin to reveal nigrostriatal defects as phenotypically measured by the pole test, which could be rescued by L-DOPA 


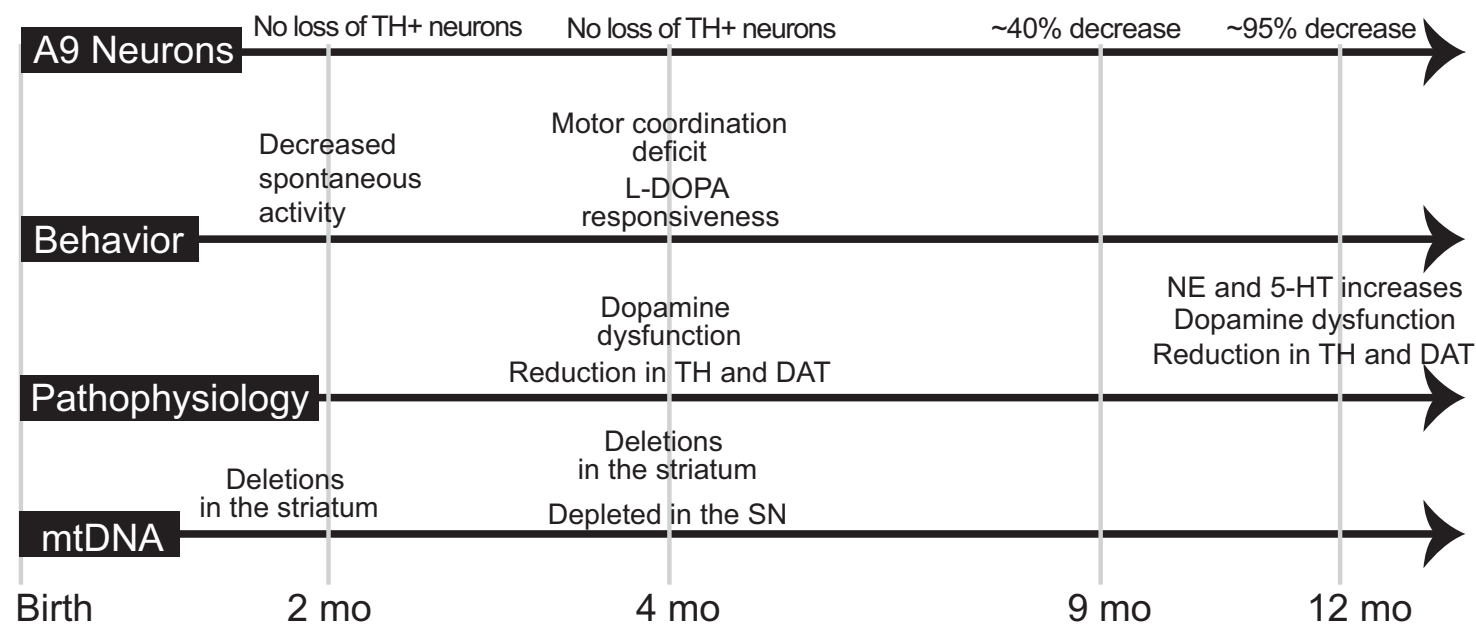

Figure 7. Timeline of phenotype, neurodegeneration, pathophysiology, and mtDNA changes during the lifetime of PD-mito-Pstl mice. Shown is a graphical representation of our findings with the PD-mito-Pstl mouse over the course of the animal's lifespan.

treatment. They also showed decreases in TH, dopamine, and DAT in the striatum. These defects preceded SN tyrosine hydroxylasepositive neuron decline, as this loss in the PD-mito-PstI mice occurred later in life ( $\sim 9$ months) with an approximate $60 \%$ decrease. At $>12$ months of age, PD-mito-PstI mice have very few THpositive neurons in the $\mathrm{SN}$ with a persistent decrease in dopamine metabolites; however, increases in NE and 5-HT were seen in the striatum that may have contributed to the relatively mild phenotype. At this point, it is unclear whether the increase in these metabolites is related solely to increased production by the remaining projecting nigral neurons or whether non-nigral projections participate. Although serotonin could originate from other regions, NE production is expected to be restricted to $\mathrm{TH}$-containing neurons.

In our transgenic PD-mito-PstI colony, we have noted no premature death of the PD-mito-PstI mouse up to 18 months. The complete depletion of dopamine in the striatum is expected to cause severe hypoactivity and death due to the lack of feeding or drinking behavior (Zhou and Palmiter, 1995); therefore, we speculate that our PD-mito-PstI mice survived because dopamine was not completely depleted even at 12 months of age (Fig. $5 B)$. Although we observed massive loss of tyrosine hydroxylasepositive neurons at this time point, there probably were sufficient numbers of dopaminergic neurons, producing enough dopamine for survival.

Another possibility to this relatively mild phenotype is that the $\mathrm{NE}$ and 5-HT changes observed in PD-mito-PstI mice after massive dopaminergic neurodegeneration may provide a certain degree of functional compensation. Although PD is often defined by the loss of A9 dopaminergic neuronal population of SN region, it is also characterized by a degeneration of the NE neuronal populations in the locus ceruleus (LC) (German et al., 1992). In addition, pharmacological MPTP PD models show increased motor disturbances and exacerbated PD pathophysiology when LC neurons are also lesioned (Mavridis et al., 1991; Marien et al., 1993). These results imply the importance of NE neurotransmission in PD and dopamine circuitry. Although 5-HT is associated with a different biosynthetic pathway, 5-HT receptors and endogenous 5-HT have been also been described to modulate striatal dopamine release (Besson et al., 1969; Navailles and De Deurwaerdère, 2011).

In human $\mathrm{PD}$, cardinal symptoms only appear after a $70-80 \%$ decrease in striatal dopamine levels, so it is reasonable to suspect plasticity and a possible compensation of other circuitry during degeneration prevents motor deficits before this threshold. This putative compensation of NE in the striatum at 12 months of age in our model is compatible with the fact that we did not detect a further decrease or loss of TH at this age (Fig. 4C,D), as TH would be needed to produce this NE increase. This may also explain why we did not observe an even greater reduction in dopamine levels in the striatum from the 4 months to $>12$ months time point, even though SN neurons were markedly reduced at $>12$ months (Figs. 3E, $5 A, B$ ).

One of the pathogenic hallmarks of PD is the presence of eosinophilic inclusions called Lewy bodies in the surviving neurons. These inclusions are immunopositive for different markers, including $\alpha$-synuclein and ubiquitin. We performed immunohistochemistry studies in brains of 4- and 12-month-old PDmito-PstI animals, using antibodies against $\alpha$-synuclein and ubiquitin, but we did not detect any positive inclusions (data not shown). Although this is a negative result, it suggests that Lewy body-like inclusions are not prominent features in our model. However, there are several well established genetic mice models, including mouse models overexpressing human or mouse $\alpha$-synuclein that do not show Lewy bodies (Magen and Chesselet, 2010) or mouse models that show inclusions that are negative for $\alpha$-synuclein (Ekstrand et al., 2007). Moreover, the presence of Lewy bodies and $\alpha$-synuclein inclusions appear also in other neurodegenerative diseases, such as multiple system atrophy and Lewy body dementia, accumulating in the brainstem and cortex and not necessarily associated with dopaminergic neurodegeneration (Papka et al., 1998; Goedert et al., 2001).

\section{Striatal dysfunctions and nigral somata demise}

The PD-mito-Pst mice showed a decrease in TH, DAT, and dopamine in the striatum at 4 months of age when there was no significant $\mathrm{TH}+$ cell body loss in the SN. On the other hand, systemic rotenone and MPTP PD pharmacological models showed early degeneration of both nigra neurons and axonal projections (Betarbet et al., 2000; Song and Haber, 2000). Another mitochondrial-based PD mouse model "mito-Park" with the genetic disruption of mitochondrial transcription factor A (TFAM) showed that although impaired dopamine release coincided with mitochondrial dysfunction (Good et al., 2011), axonal degeneration was related to cell body loss (Ekstrand et al., 2007). 
To our knowledge, the mouse models of PD described above have not shown that striatal dysfunction leads to behavioral phenotypes preceding substantial SN dopaminergic cell body loss.

We observed a decrease in the presynaptic DAT at 4 and $>12$ months of age. Although dopamine and TH depletion in the striatum could be due solely to the loss of dopaminergic axonal projections in the striatum, our finding that there was a nonproportional decrease in DA catabolites (e.g., DOPAC) suggests that DA metabolism is altered by the bioenergetics defect before cell body loss.

Our model has the benefit of having a relatively slow OXPHOS impairment, progressive loss of dopaminergic neurons in the SN and a lesser effect on VTA neurons even at later stages, mimicking the pattern seen in PD. This slow degeneration can possibly explain the generally mild phenotype. Our previous work with mito-PstI under the CaMKII $\alpha$ promoter showed that although mito-PstI was expressed in the same CNS regions, the phenotype differed due to the onset severity of OXPHOS and speed of neurodegeneration (Fukui and Moraes, 2009; Pickrell et al., 2011). The "MitoPark" mouse showed a massive $40 \%$ decrease in SN neurons at 3 months of age with an additional $20 \%$ decrease 1 month later (Ekstrand et al., 2007). In PD-mito-PstI model, a $60 \% \mathrm{TH}+\mathrm{SN}$ neuronal loss did not occur until 9 months of age (Fig. $3 B, D$ ), so the delay in neuron loss caused by a mild bioenergetic defect makes this mouse model useful to understand the biochemical mechanisms preceding dopaminergic neuron death.

There are clear intrinsic differences in the susceptibility to mitochondrial defects in the $\mathrm{SN}$ region compared to the VTA. In our model, even after an almost complete ablation of the SN neurons at 12 months, we observed abundant neurons in the VTA region (Fig. $3 E$ ). Other groups have also reported this increased resistance of the VTA region in other mouse models driven by either the DAT-Cre "MitoPark mouse" or the TH-Credriven succinate dehydrogenase complex II conditional knockout (Ekstrand et al., 2007; Díaz Castro et al., 2010). Microarray studies on microdissected neurons from the SN and VTA region revealed differences in gene expression related to mitochondrial metabolism, which may help explain the increased sensitivity of the $\mathrm{SN}$ region to mitochondrial insults (Greene et al., 2005). In summary, the PD-mito-PstI model mimics the pattern of SN $\mathrm{TH}+$ neuronal loss observed in the human disease, where the relative resistance of VTA neurons may be due to their intrinsic mitochondrial properties.

\section{Mitochondrial OXPHOS dysfunction and PD}

Because mitochondrial dysfunction has been closely associated with the pathophysiology of $\mathrm{PD}$, we believe our PD-mito-PstI model provides important clues to the pathophysiology of the disease. Due to the reduction of mitochondria gene products in postmortem PD human brains, therapeutic interventions overexpressing peroxisome proliferator-activated receptor gamma coactivator 1- $\alpha$ (PGC- $\alpha$ ), a coactivator that stimulated mitochondrial biogenesis, is extremely attractive and proven to work in cell culture models overexpressing $\alpha$-synuclein and rotenonetreated primary midbrain cultures (Zheng et al., 2010). Coenzyme Q10, which shuttles electrons from complexes I and II to complex III, has been shown to slow the progression of the disease with phase III trials in progress (Shults et al., 2002). Our laboratory found that increased mitochondrial biogenesis preserved muscle function in sarcopenia and mitochondrial myopathy models (Wenz et al., 2008, 2009). Our PD transgenic mouse model provides a valuable tool to test new mitochondrial-based therapies for PD.

In summary, we have created a novel mouse model of parkinsonism associated with a chronic damage to mtDNA, a feature previously associated with PD. Pathophysiological studies showed that axonal dysfunction of the A9 neurons contributes to behavioral phenotypes in the PD-mito-PstI model. This mouse model recapitulates A9 dopaminergic neuronal loss, striatal dysfunction, monoamine neurotransmitter defects, late onset, and loss of locomotive functions reversed by L-DOPA treatment, all of which are components of human PD. Our results showed that motor phenotypes in the PD-mito-PstI mouse model initially arise from a striatal dysfunction and not A9 cell body loss. We also have observed that by affecting only the dopaminergic circuitry, PD-mito-PstI mice show changes in noradrenergic and serotonin neurotransmitters later in life with possibly compensatory functions.

\section{References}

Anderson S, Bankier AT, Barrell BG, de Bruijn MH, Coulson AR, Drouin J, Eperon IC, Nierlich DP, Roe BA, Sanger F, Schreier PH, Smith AJ, Staden R, Young IG (1981) Sequence and organization of the human mitochondrial genome. Nature 290:457-465.

Bender A, Krishnan KJ, Morris CM, Taylor GA, Reeve AK, Perry RH, Jaros E, Hersheson JS, Betts J, Klopstock T, Taylor RW, Turnbull DM (2006) High levels of mitochondrial DNA deletions in substantia nigra neurons in aging and Parkinson disease. Nat Genet 38:515-517.

Besson MJ, Cheramy A, Feltz P, Glowinski J (1969) Release of newly synthesized dopamine from dopamine-containing terminals in the striatum of the rat. Proc Natl Acad Sci U S A 62:741-748.

Betarbet R, Sherer TB, MacKenzie G, Garcia-Osuna M, Panov AV, Greenamyre JT (2000) Chronic systemic pesticide exposure reproduces features of Parkinson's disease. Nat Neurosci 3:1301-1306.

Cagniard B, Beeler JA, Britt JP, McGehee DS, Marinelli M, Zhuang X (2006) Dopamine scales performance in the absence of new learning. Neuron 51:541-547.

Díaz Castro B, García-Flores GP, Pintado O, Rodríguez-Gómez JA, LópezBareno J, Piruat JI (2010) Early postnatal catecholaminergic neural loss in a mouse model genetically deficient in mitochondrial complex II. Soc Neurosci Abstr 36:52.13.

Ekstrand MI, Terzioglu M, Galter D, Zhu S, Hofstetter C, Lindqvist E, Thams S, Bergstrand A, Hansson FS, Trifunovic A, Hoffer B, Cullheim S, Mohammed AH, Olson L, Larsson NG (2007) Progressive parkinsonism in mice with respiratory-chain-deficient dopamine neurons. Proc Natl Acad Sci U S A 104:1325-1330.

Fukui H, Moraes CT (2009) Mechanisms of formation and accumulation of mitochondrial DNA deletions in aging neurons. Hum Mol Genet 18:1028-1036.

German DC, Manaye KF, White CL 3rd, Woodward DJ, McIntire DD, Smith WK, Kalaria RN, Mann DM (1992) Disease-specific patterns of locus coeruleus cell loss. Ann Neurol 32:667-676.

Goedert M, Jakes R, Anthony Crowther R, Grazia Spillantini M (2001) Parkinson's disease, dementia with Lewy bodies, and multiple system atrophy as $\alpha$-synucleinopathies. Methods Mol Med 62:33-59.

Good CH, Hoffman AF, Hoffer BJ, Chefer VI, Shippenberg TS, Bäckman CM, Larsson NG, Olson L, Gellhaar S, Galter D, Lupica CR (2011) Impaired nigrostriatal function precedes behavioral deficits in a genetic mitochondrial model of Parkinson's disease. FASEB J 25:1333-1344.

Greene JG, Dingledine R, Greenamyre JT (2005) Gene expression profiling of rat midbrain dopamine neurons: implications for selective vulnerability in parkinsonism. Neurobiol Dis 18:19-31.

Hattingen E, Magerkurth J, Pilatus U, Mozer A, Seifried C, Steinmetz H, Zanella F, Hilker R (2009) Phosphorus and proton magnetic resonance spectroscopy demonstrates mitochondrial dysfunction in early and advanced Parkinson's disease. Brain 132:3285-3297.

Kraytsberg Y, Kudryavtseva E, McKee AC, Geula C, Kowall NW, Khrapko K (2006) Mitochondrial DNA deletions are abundant and cause functional impairment in aged human substantia nigra neurons. Nat Genet 38:518-520. 
Magen I, Chesselet MF (2010) Genetic mouse models of Parkinson's disease: the state of the art. Prog Brain Res 184:53-87.

Marien M, Briley M, Colpaert F (1993) Noradrenaline depletion exacerbates MPTP-induced striatal dopamine loss in mice. Eur J Pharmacol 236:487-489.

Matsuura K, Kabuto H, Makino H, Ogawa N (1997) Pole test is a useful method for evaluating the mouse movement disorder caused by striatal dopamine depletion. J Neurosci Methods 73:45-48.

Mavridis M, Degryse AD, Lategan AJ, Marien MR, Colpaert FC (1991) Effects of locus coeruleus lesions on parkinsonian signs, striatal dopamine and substantia nigra cell loss after 1-methyl-4-phenyl-1,2,3,6tetrahydropyridine in monkeys: a possible role for the locus coeruleus in the progression of Parkinson's disease. Neuroscience 41:507-523.

Meredith GE, Kang UJ (2006) Behavioral models of Parkinson's disease in rodents: a new look at an old problem. Mov Disord 21:1595-1606.

Navailles S, De Deurwaerdère P (2011) Presynaptic control of serotonin on striatal dopamine function. Psychopharmacology (Berl) 213:213-242.

Papka M, Rubio A, Schiffer RB (1998) A review of Lewy body disease, an emerging concept of cortical dementia. J Neuropsychiatry Clin Neurosci $10: 267-279$.

Pickrell AM, Fukui H, Wang X, Pinto M, Moraes CT (2011) The striatum is highly susceptible to mitochondrial oxidative phosphorylation dysfunctions. J Neurosci 31:9895-9904.

Reeve AK, Krishnan KJ, Elson JL, Morris CM, Bender A, Lightowlers RN, Turnbull DM (2008) Nature of mitochondrial DNA deletions in substantia nigra neurons. Am J Hum Genet 82:228-235.

Schapira AHV, Cooper JM, Dexter D, Clark JB, Jenner P, Marsden CD (1990a) Mitochondrial complex I deficiency in Parkinson's disease. J Neurochem 54:823-827.

Schapira AHV, Mann VM, Cooper JM, Dexter D, Daniel SE, Jenner P, Clark JB, Marsden CD (1990b) Anatomic and disease specificity of NADH CoQ 1 reductase (complex I) deficiency in Parkinson's disease. J Neurochem 55:2142-2145.

Schmittgen TD, Livak KJ (2008) Analyzing real-time PCR data by the comparative CT method. Nat Protoc 3:1101-1108.
Shimohama S, Sawada H, Kitamura Y, Taniguchi T (2003) Disease model: Parkinson's disease. Trends Mol Med 9:360-365.

Shults CW, Oakes D, Kieburtz K, Beal MF, Haas R, Plumb S, Juncos JL, Nutt J, Shoulson I, Carter J, Kompoliti K, Perlmutter JS, Reich S, Stern M, Watts RL, Kurlan R, Molho E, Harrison M, Lew M (2002) Effects of coenzyme Q10 in early Parkinson disease: evidence of slowing of the functional decline. Arch Neurol 59:1541-1550.

Song DD, Haber SN (2000) Striatal responses to partial dopaminergic lesion: evidence for compensatory sprouting. J Neurosci 20:5102-5114.

Srivastava S, Moraes CT (2001) Manipulating mitochondrial DNA heteroplasmy by a mitochondrially targeted restriction endonuclease. Hum $\mathrm{Mol}$ Genet 10:3093-3099.

Srivastava S, Moraes CT (2005) Double-strand breaks of mouse muscle mtDNA promote large deletions similar to multiple mtDNA deletions in humans. Hum Mol Genet 14:893-902.

Vyas I, Heikkila RE, Nicklas WJ (1986) Studies on the neurotoxicity of 1-methyl-4-phenyl-1,2,3,6-tetrahydropyridine: inhibition of NAD-linked substrate oxidation by its metabolite, 1-methyl-4-phenylpyridinium. J Neurochem 46:1501-1507.

Wenz T, Diaz F, Spiegelman BM, Moraes CT (2008) Activation of the PPAR/PGC-1alpha pathway prevents a bioenergetic deficit and effectively improves a mitochondrial myopathy phenotype. Cell Metab 8:249-256.

Wenz T, Rossi SG, Rotundo RL, Spiegelman BM, Moraes CT (2009) Increased muscle PGC-1alpha expression protects from sarcopenia and metabolic disease during aging. Proc Natl Acad Sci U S A 106:2040520410.

Zheng B, Liao Z, Locascio JJ, Lesniak KA, Roderick SS, Watt ML, Eklund AC, Zhang-James Y, Kim PD, Hauser MA, Grünblatt E, Moran LB, Mandel SA, Riederer P, Miller RM, Federoff HJ, Wüllner U, Papapetropoulos S, Youdim MB, Cantuti-Castelvetri I, et al. (2010) PGC-1, a potential therapeutic target for early intervention in Parkinson's disease. Sci Transl Med 2:52ra73.

Zhou Q-Y, Palmiter RD (1995) Dopamine-deficient mice are severely hypoactive, adipsic, and aphagic. Cell 83:1197-1209. 